\title{
Horseback riding therapy for a deafblind individual enabled by a haptic interface
}

\author{
Matjaž Ogrinc ${ }^{1,2}$, Ildar Farkhatdinov ${ }^{1,3}$, Rich Walker $^{2}$, \\ Etienne Burdet ${ }^{1}$ \\ ${ }^{1}$ Department of Bioengineering, Imperial College of Science, Technology and Medicine, \\ London SW72AZ, United Kingdom, \\ \{matjaz.ogrinc, i.farkhatdinov, e.burdet\}@imperial.ac.uk \\ ${ }^{2}$ Shadow Robot Company LTD., \\ London N11LX, United Kingdom \\ $\{$ matjaz,rw\}@shadowrobot.com \\ ${ }^{3}$ School of Electronic Engineering and Computer Science, \\ Queen Mary University of London, London E14NS, United Kingdom \\ i.farkhatdinov@qmul.ac.uk
}

\begin{abstract}
We present a haptic interface to help deafblind people to practice horse riding as a recreational and therapeutic activity. Horseback riding is a form of therapy which can improve self-esteem and sensation of independence. It has been shown to benefit people with various medical conditions including autism. However, in the case of deafblind riders an interpreter must stand by at all times to communicate with the rider by touch. We developed a simple interface that enables deafblind people to enjoy horseback riding while the instructor is remotely providing cues, which improves their independence. Experiments demonstrated that an autistic deafblind individual exhibits similar responses to navigational cues as an unimpaired rider. Motivation is an important factor in therapy and is frequently determinant of its outcome, therefore the user attitude towards the therapy methods is key. The answers to questionnaires filled by the rider, family and the instructor show that our technique gives the rider a greater sense of independence and more joy compared to standard riding where the instructor is walking along with the horse.
\end{abstract}

Keywords: vibrotactile $\cdot$ autism $\cdot$ navigation $\cdot$ deafblind 


\section{Introduction}

Animal assisted therapy (AAT) is used to improve physical and mental health of people with various conditions, including individuals suffering from dementia (Richeson, 2003) and Alzheimers (Edwards \& Beck, 2002), as well as children with attention deficit and hyperactivity disorder (ADHD) (Busch et al., 2016). AAT and horseback riding in particular resulted in significant improvements in behavioral and social interaction in individuals affected by autism spectrum disorder (ASD) (Bass, Duchowny, \& Llabre, 2009; O'Haire, 2013; Sams, Fortney, \& Willenbring, 2006). It has also been found that riding improves physical strength and posture, and commanding such a large animal that responds to one's cues also improves self-esteem (Fine, 2010). Though it may be beneficial for some deafblind individuals as well, their sensory loss can prevent them from fully benefiting from therapeutic horseback riding. We developed a tactile interface to facilitate remote communication between deafblind users and the riding instructor. Due to its simplicity and low cost, the device could also be used for blind riders as an alternative to auditory cues delivered via a headset. We hypothesized that the deafblind rider is able to interpret and respond to the tactile cues similarly to a healthy user. We tested this by comparing their arm pulling motion in response to cues captured using inertial measurement units (IMUs). Secondly, we hypothesized that the introduction of remote communication brings greater feeling of independence to the user. In deafblind user studies the concept of independence is used to define the level of autonomy in life activities (Azenkot et al., 2011). However, the independence in the context of our study corresponds to the capability of the deafblind to practice horseback riding without being physically guided, as it is normally done in conventional horseback riding. We suggest that being able to ride a horse without a guide in direct proximity provides a deafblind user a sense of independence and joy. Even though this subjective perceptions may not have a direct functional value, they provide the motivation to the rider to continue the therapy and not abandon it. Motivation is an important factor in rehabilitation and is frequently determinant of rehabilitation outcome (Maclean, Pound, Wolfe, \& Rudd, 2002).

The Background section presents the conventional horseback riding therapy for the deafblind and highlights its limitations, in particular the missing sense of independence. To increase it, we suggest use of haptics as the modality most suitable to bridge the sensory limitations of the deafblind. We review the existing assistive technology for navigation and communication and provide grounds for designing our own interface. We review sensory, cognitive and mental difficulties that can be observed in many individuals with the deafblindness, such as autism, that could affect successful use of our interface. For instance, autism is known to be related to tactile hypersensitivity. In the Case study we describe the tactile interface in detail, provide quantitative and qualitative methodology and the results of the study. Conclusions and limitations are discussed in the final section.

Acknowledgment: This work is supported by the European Commission under EU-FP7 grants PITNGA-2012-317488-CONTEST, ICT-601003 BALANCE, ICT-2013-10 SYMBITRON and H2020 ICT 644727

COGIMON. We would like to thank Doug Smith from RDA for fruitful discussions and assistance during the tests. 


\section{Background}

\section{Deafblind horseback riding}

The World Health Organization reports the world's blind population is about 39 million (World Health Organisation, 2014), while there are around 50,000 deafblind individuals in USA alone (Caporusso, Trizio, \& Perrone, 2014) and 150,000 in Europe (Drescher, 2006). Due to the combination of both sensory impairments, deafblind people are unable to successfully navigate without touch. In the conventional way of deafblind horse riding, the rider either passively rides the horse as it is being guided by the instructor, or the rider controls the reins while the instructor communicates riding cues by touch. For instance, predefined cues can suggest the rider commands "go", "stop", "turn left" and "turn right". However, such approach is very limited. Because the rider cannot experience independent control and needs immediate presence of a therapist, this conventional therapy cannot produce maximal positive therapeutic effect on the rider, who feels strongly dependent on the instructor. Therefore we propose a system that would allow remote communication between the instructor and the rider. This can only be achieved by using the haptic modality, or the sense of touch. In the proposed concept illustrated in Fig. 1a, a therapist/instructor sends the navigational commands to a deafblind rider remotely.

\section{Communication aids for blind and deafblind}

Development of novel technologies for blind and deafblind users is crucial as it can enable them to take part in various activities. In recent years, haptic interfaces have been developed that enable remote communication for the deafblind. For instance, the Lorm glove interface enables input and output using the Lorm alphabet (Gollner, Bieling, \& Joost, 2012), and the $d b G L O V E$ enables bidirectional communication based on the Malossi alphabet (Caporusso, 2008). Some lingual communication devices for the blind, most commonly Braille interfaces, and devices for spatial orientation, can also be used by the deafblind (Caporusso et al., 2014). A survey presented in (Dakopoulos \& Bourbakis, 2010) reviews a number of devices for the deafblind, ranging from sensory substitution devices that present visual information through another modality, orientation aids and position locator devices. For example, the vibrotactile belt from (Nagel, Carl, Kringe, Märtin, \& König, 2005) can deliver directional information by indicating the direction to the target location, acting as a vibrotactile compass. A similar device presented in (Tsukada \& Yasumura, 2004) also employs GPS signals to facilitate way-point navigation. In general, these interfaces rely on satellite localization for outdoor applications, beacons for indoor navigation and various position sensors and cameras for obstacle detection. An interesting concept of remote guidance has been presented in (Scheggi, Talarico, \& Prattichizzo, 2014). The system is based on transferring video stream from camera glasses worn by the disabled to a remote assistant, who then issues navigation cues via tactile stimuli. However, to our knowledge there is no low cost commercial solution for spatial navigation that provides a simple input interface and can be easily used by a deafblind rider and a riding instructor. Therefore, we developed a haptic communication system giving a deafblind person the ability to command a horse independently, rather than through physical guidance of the instructor. This paper presents this interface and analyses its use. Fig. 1b shows a deafblind rider using 


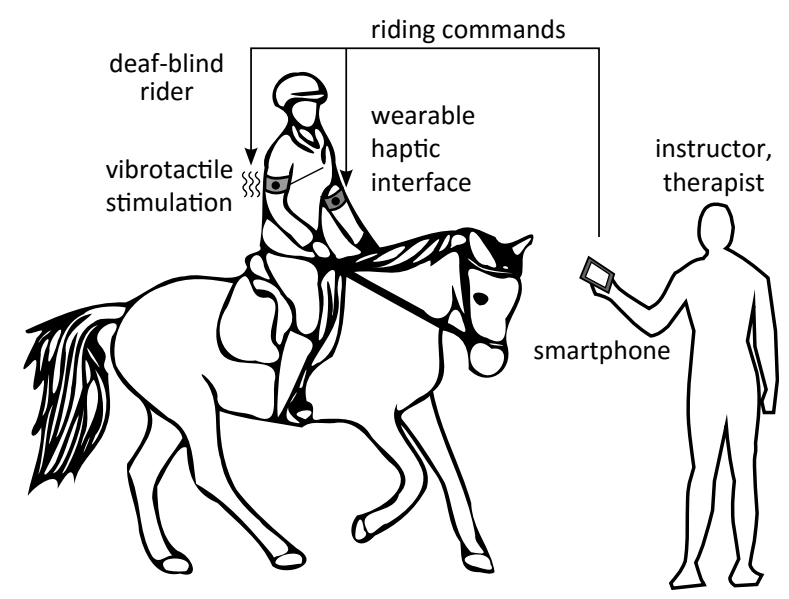

(a)

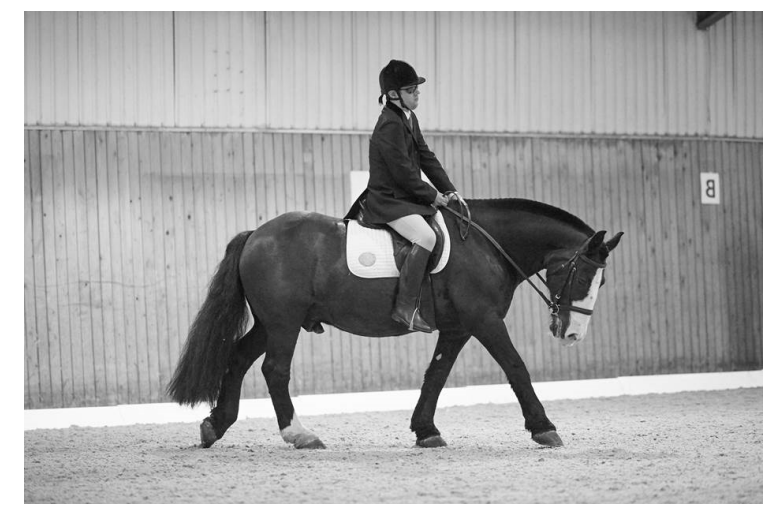

(b)

Figure 1. Deafblind horseback riding. The instructor communicates messages to the rider remotely using the proposed wireless interface. (a) The overall concept. (b) A photo of the deafblind rider during the UK's National Competition in Riding for the Disabled

the proposed device. The detailed technical description of the apparatus is provided in the Case study section.

\section{Deafblindness and related disorders}

Depending on the age of onset of sensory loss, deafblindness can be congenital or acquired. In the case of the former the impairment occurs before age of two and is also known as prelingual deafblindness. Only around one in five cases of deafblindess is congenital. The common causes are CHARGE syndrome and prematurity (Dammeyer, 2012). On the other hand, the most common cause of acquired deafblindness is an extremely rare genetic disorder known as Usher Syndrome. This is the case in approximately half of the people with the impairment, excluding the cases related to aging (Moller, 2003). An accurate identification of congenital impairment is difficult as it requires cooperation of the examined person at 
a very young age who may also be affected by severe motor, cognitive and behavioral impairments.

The barriers in communication and social interaction caused by deafblindness can lead to a number of health-related difficulties, including depression, cognitive decline, developmental disorder in children and psychological distress (Dammeyer, 2014). Deafblindness also affects the ability to achieve autonomous living, independent mobility and social inclusion. In one study it has been found that $74 \%$ of congenitally deafblind people had a mental and/or behavioral disorder diagnose (Dammeyer, 2011). Autism or autism-related conditions are common among blind children (Cass, 1998) and children with hearing loss (Carvill, 2001). Autism spectrum disorder (ASD) is a pervasive developmental disorder commonly exhibited in early childhood. It causes severe and often lifelong effects on communication, socialization, and tendencies toward restricted interests or repetitive behaviors (American Psychiatric Association, 2000). Sensory-perceptual anomalies occur in approximately $70 \%$ of cases (Baranek, David, Poe, Stone, \& Watson, 2006) in multiple forms and across various modalities (Kern et al., 2006). Some individuals exhibit hyper sensitivity while other show hypo sensitivity. Though hyper sensitivity can cause unpleasant perception of a simple touch (a hug, particular textile or food texture), hypo sensitivity can have more severe consequences. For instance, hypo sensitivity to pain can lead to serious injuries that would have normally been prevented in response to a noxious sensation. Both hypo- and hypersensitivity can occur in the same individuals. Some individuals with ASD may not have any sensory issues while others may experience extreme levels of sensory overload that may prevent social interactions. Blakemore et al. reported altered detection of vibration stimuli in both adults and children with ASD (Blakemore et al., 2006). However, other studies report normal tactile detection in autism (Cascio et al., 2008; Güçlü, Tanidir, Mukaddes, \& Ünal, 2007). Furthermore, it remains unclear whether the different responses to stimulation in ASD are emotional, or a consequence of altered sensory mechanisms (Puts, Wodka, Tommerdahl, Mostofsky, \& Edden, 2014). Because of typical sensory abnormalities in ASD, the design of a vibrotactile display for the deafblind has to be considered carefully. However, despite these sensory abnormalities, vibrotactile interfaces have previously been used with ASD individuals in order to improve social interaction (Changeon, Graeff, Anastassova, \& Lozada, 2012; Tang, McMahan, \& Allen, 2014). Tang et al. developed a tactile sleeve with an array of motors that can display simple and dynamic stimuli in order to simulate touch in social situations (Tang et al., 2014) and practice social interactions. Changeon et al. developed a vibrotactile game controller for transmitting emotional messages to children with autism while they engage in a videogame.

Another sensory disorder that can be related to deafblindess and is relevant in horseback riding is impairment of the vestibular sense (Kaga, Shinjo, Jin, \& Takegoshi, 2008). Congenitally deaf infants and children commonly suffer vestibular failure in both ears, and impairment of postural control, locomotion, and gait. Abnormalities of the vestibular sense in ASD are shown in abnormal posturing, balance, and eye movements (Kern et al., 2007). Thus, horseback riding therapy may not be appropriate for some blind, deaf or deafblind individuals. 


\section{Case study with an autistic deafblind rider}

\section{The vibrotactile interface}

The interface is composed of inexpensive, simple and robust components. The overall control flow of the system is illustrated in Fig. 2. Two vibration motors (model 307-100, Precision Microdrives, UK) were selected to display haptic cues to a deafblind rider. The actuators are encapsulated in a robust 3D printed plastic case and attached to wearable textile straps for convenience of attachment. According to the manufacturer data sheet, the rise and stop times for the selected motors are 34 and $73 \mathrm{~ms}$ respectively, which is sufficient for horse riding in which execution of the commands usually takes more than a second. The motors are powered by a custom-designed control module, which is small enough to be carried in a rider's pocket. The control module contains a microcontroller (Arduino Pro Mini) and wireless communication electronics, and is connected to a host mobile device via Bluetooth communication protocol using the RN41 bluetooth module (Roving Networks Inc). The module and the built in antenna are designed for range up to 100 meters. The device is powered by a $3.7 \mathrm{~V} \mathrm{LiPO}$ rechargeable battery. The MOSFET transistors operating as switches amplify the PWM signals generated by the microcontroller. The data exchange with the host device was implemented with the standard serial communication protocol at 38400 baud rate. The interface hardware (without Android mobile device) costs under $£ 50$.

An Android OS application has been developed for controlling the interface. The graphical user interface of the application consists of buttons which trigger the basic instructions described above. Vibrations with the right or left motor suggest a turn in the respective direction. "go" is represented by a short vibration pulse with both motors, and "stop" by a long simultaneous stimulation of both motors. The application also allows to set the vibration intensity to a level that is comfortable for the rider. All user input events were logged by the application for further analysis with one millisecond resolution.

\section{Methods}

Subjects and experimental protocol. The subject examined in this case study was 31 years old at the time of the trials. He was profoundly deaf and communicating in British sign language (BSL) before becoming blind at the age of 10 . The cause of his blindness is retinopathy of prematurity. He was diagnosed at the age of 13 with Aspergers, a form of high functioning autism. According to his family, he has never been averting touch or finding textures unpleasant, except for some food textures, and has no particular aversion to vibrations. He has been using Braille for most of his life, including modern dynamic Braille displays, therefore is well accustomed to dynamic tactile stimulation. He is also enjoying pottery. He practiced horseback riding as a child, but was forced to abandon it with the loss of sight and has recently resumed the activity. He practiced riding for three months before switching to the present haptic interface. He had been using the interface for 15 months at the time of the trials of this study.

A female (age 22) subject without disability also participated in the study as control subject. She has been riding since childhood and has prior but limited experience with our haptic interface as she took part in a preliminary trial several months prior when she used 
the interface for around 30 minutes. During the trials she was blindfolded, wore ear plugs and noise cancellation headphones.

The tests were carried out in a riding arena, the same horse was used by both subjects. The subject wore our tactile interface which was remotely controlled via a smart phone by a professional riding coach. The participants received all required information on the tactile mapping commands and carried out familiarization trials. A deafblind tactile language interpreter facilitated communication with the disabled subject. In the riding tests the subjects were asked to follow semi-random sets of commands given by the instructor. The sequence of the commands depended on the position of the horse. Though the riding arena measures $20 \times 50 \mathrm{~m}$ and is within range of the tactile interface, the range of motion capture equipment was smaller and therefore only half of the arena was used. This somewhat limits the maneuverability of the horse and therefore a truly random turn sequence was not always possible.

We conducted interviews with the deafblind rider, his family and his instructor to investigate the long term effects of practicing horseback riding and the benefits of using the remote haptic communication as compared to conventional communication. A permission from the family of the blind-deaf rider was obtained, as approved per an ethical agreement

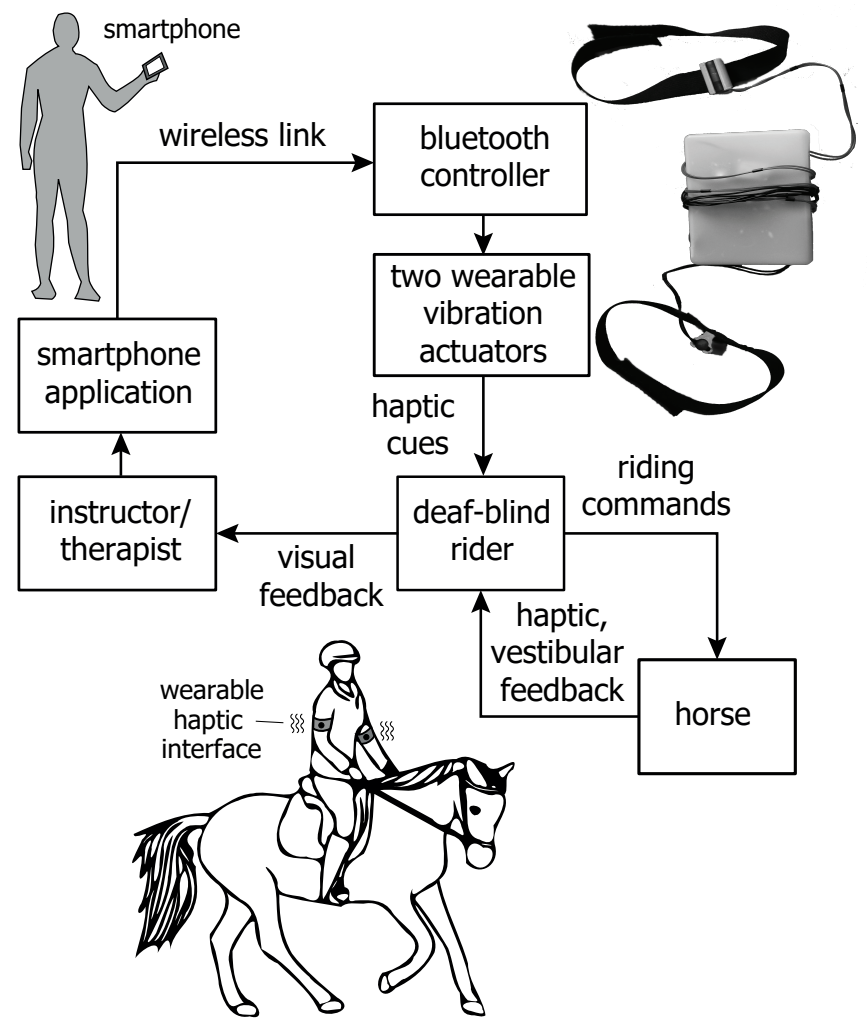

Figure 2. Interface's main components and functionality. An instructor sends the riding commands via smart phone application and looks at the riding; the commands are displayed to a deafblind rider with haptic cues; a rider feels the horse movements via haptic and vestibular sensory cues. 


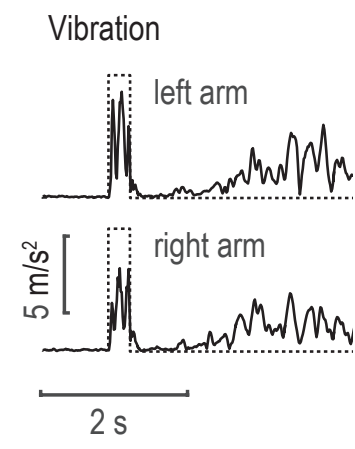

(a)

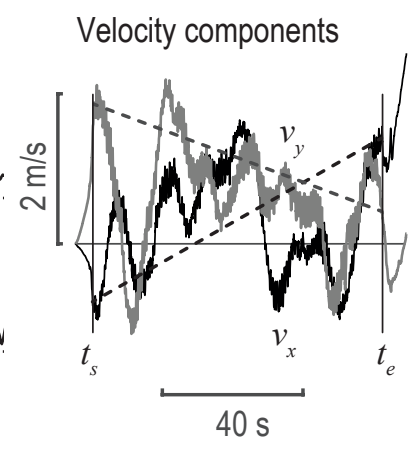

(b)

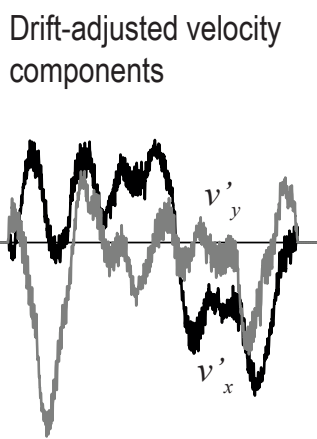

(c)
Drift-adjusted velocity

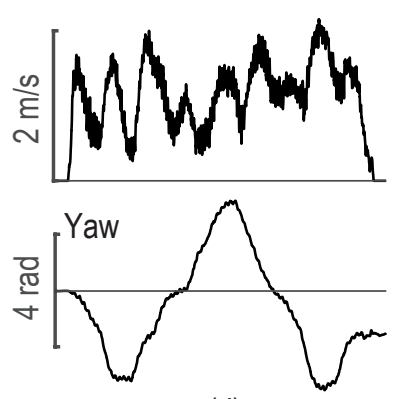

(d)

Figure 3. Synchronization of motion capture data with recorded occurrences of stimulation and reconstruction of motion trajectory. (a) Alignment of the IMU signals and riding command signals (dotted line). The vibration (solid line) of the initial "go" command is detected by IMUs mounted on top of the stimulators. (b-d) The trajectory estimation: Integrated accelerometer output (velocity), velocity after drift compensation, magnitude of the velocity and yaw angle.

letter (Ethics, health and safety consent) from Riding for Disabled Association (RDA) charity.

Quantitative evaluation with motion capture. Three XSens MTw (Enschede, Netherlands) inertial measurement units (IMU) were employed to track the motion of the rider and their arms. The modules transmitted the data wirelessly to a receiver device connected to a laptop PC via USB at the rate of $75 \mathrm{~Hz}$. The receiver and the laptop were positioned at the side of the riding arena. The said system's declared range is 20-50 meters. One module was attached to a subject's torso to track the riding motion. Additional two IMUs were attached to the subjects' upper arms on top of the vibration actuators. This enabled us to detect the occurrence of vibrations and thus synchronize the motion data to the stimulation occurrences. We were also able to observe the riders' pulling motion of their arms in response to the cues.

The smartphone application stores the stimulation cues timestamps into a log file. As the smartphone is not connected to the motion capture system in real time, the data must be synchronized offline. For this reason, the riders were wearing IMUs on their arms, mounted on top of the actuators in order to pick up the vibrations. The first "go" stimulus was then manually aligned to IMU signals. Fig. 3a shows the accelerometer (continuous) and stimulation (dotted) time series after synchronization.

The XSens MTx IMU measured translational acceleration, angular velocity and magnetic field, which after internal fusion provided orientation in roll-pitch-yaw form and acceleration free of the gravity component. It is common practice to obtain the position by double integration of acceleration. However, the bias in the noise present in acceleration measurement introduces drift in the velocity with the integration. This is shown in Fig. 3b. As the initial and final velocities of each trial at $t_{s}$ and $t_{e}$ respectively should be zero, we can compensate for this phenomenon. To obtain the drift-free velocity $\boldsymbol{v}^{\prime}$ we subtract the estimated drift (dotted black lines). Fig.3c shows the horizontal components of the velocity 
vector $\boldsymbol{v}^{\prime}$. This procedure of drift compensation is commonly used in gait analysis, where the compensation is executed at each step to correct the acceleration measurement during the swing phase (Sabatini, Martelloni, Scapellato, \& Cavallo, 2005). Finally, the position vector is obtained by integration of velocity $\boldsymbol{p}(k)=f_{s}^{-1} \sum_{0}^{k}\left\|\boldsymbol{v}^{\prime}(k)\right\|[\cos (\gamma(k)), \sin (\gamma(k))]^{T}$, where $f_{s}$ is sampling rate, $\gamma(k)$ is measured yaw angle, and time at sample $k$ is $t(k)=k / f_{s}$. Fig. 3d shows the velocity and (yaw) orientation.

During pulling of the reins, the rider's upper arms move backwards in the sagittal plane as a result of shoulder extension and elbow flexion. The former is reflected in the pitch angles measured by the IMUs on rider's upper arms. The pitch angles were differentiated to obtain the rate of change, and low-pass filtered at $1 \mathrm{~Hz}$ using a 2nd order Butterworth filter. Riders' pulling actions can be thus observed in response to tactile cues.

Qualitative evaluation. The deafblind rider, his family and riding instructor Were interviewed after the trials. Our interest was in the benefits that the wireless interface (haptic communication - HC) brings relative to the conventional communication - CC during horseback riding, in terms of independence, confidence and enjoyment. We were also concerned with the rider's comfort and safety. The questions listed in Table 2 were sent by email and completed within a couple of days after the quantitative measurements. The participants of the interview gave their answers on one of the two 5-level Likert scale, as shown in Table 2. The deafblind rider filled the questionnaire with the help of a Braille computer interface. The questions addressed at the rider are concerned with his comfort, safety, enjoyment, feeling of independence and perception of obedience of the horse. The instructor was asked about his view on same aspects. As ASD is commonly associated with ADHD, questions 18-20 and 23-25 were concerned with the deafblind rider's potential attention difficulties. Though this paper is not concerned with clinical evaluation and therapy effects, the feedback from the instructor and the family provides brief insight on subject's attention span and preliminary feedback on potential therapeutic benefits.

\section{Results}

The concept of four navigation commands proved to be very simple to use. The subjects adopted the method within moments and were able to understand the cues immediately. In this section we present the results of both motion data analysis and qualitative feedback.

Riding trajectory analysis. The overall duration of recording including the setup of motion capture was 30 minutes for each subject. Several trials were carried out, however, due to practical difficulties, such as limited workspace and resulting occasional IMU signal loss, some data was discarded. Three trials were successfully recorded for the control subject and two trials for the deafblind subject. Fig. 4 shows the estimated movement trajectories for the tests with the control (Fig. $4 \mathrm{a}-\mathrm{c}$ ) and the deafblind (Fig. $4 \mathrm{~d}$, e) riders. The colored markers indicate stimulation of the right (gray) and the left (black) motors. Table 1 shows basic characteristics of the trials. The data shows similar mean speed in the deafblind and control subject, and on the straight sections both reached similar maximum speeds. The deafblind subject failed to execute some commands to change the direction of riding. Overall $53 \%$ of the commands were carried out immediately. In other words, six turns were 
executed promptly, one after two sequential cues and two after three or more repetitions. In all trials both subjects successfully executed the stop command, which is most important for safety reasons.

Table 1

\begin{tabular}{l|ccc|cc} 
subject & \multicolumn{3}{|c|}{ healthy } & \multicolumn{2}{c}{ deafblind } \\
\hline trial & $\mathrm{a}$ & $\mathrm{b}$ & $\mathrm{c}$ & $\mathrm{d}$ & $\mathrm{e}$ \\
perceived cues & $7 / 7$ & $8 / 8$ & $10 / 10$ & $9 / 9$ & $9 / 9$ \\
executed cues & $7 / 7$ & $8 / 8$ & $10 / 10$ & $7 / 9$ & $5 / 9$ \\
trial length $[\mathrm{m}]$ & 115.15 & 118.37 & 100.68 & 123.57 & 144.42 \\
trail duration $[\mathrm{s}]$ & 78.79 & 98.77 & 87.03 & 108.48 & 96.85 \\
mean speed $[\mathrm{m} / \mathrm{s}]$ & 1.46 & 1.20 & 1.16 & 1.14 & 1.49 \\
peak speed $[\mathrm{m} / \mathrm{s}]$ & 3.02 & 2.64 & 2.16 & 3.04 & 2.78
\end{tabular}

Basic parameters of the recorded trajectories.

Perception and responses to cues. The pitch angles measured by the IMUs on rider's arms were differentiated to obtain the rate of change, and filtered with a low-pass filter with a cutoff frequency of $1 \mathrm{~Hz}$. Fig. 5a depicts typical signals in response to a cue for the control subject. As the cue is given, the pitch rate peaks in the negative direction representing the pull of the reins of the corresponding arm. In response, the horse changes direction as indicated by the change in rate of yaw. Panels b and c depict two turns of

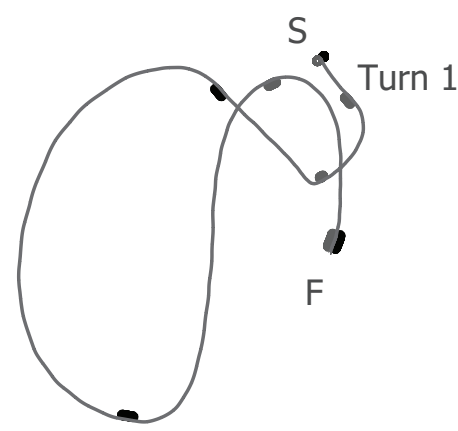

(a)

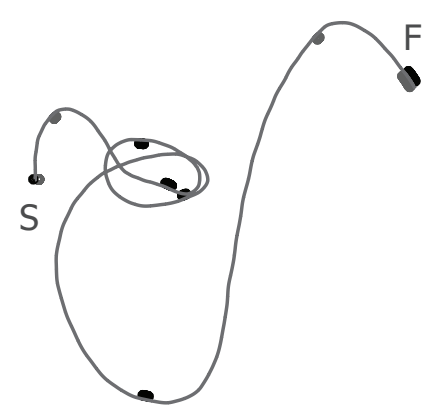

(b)

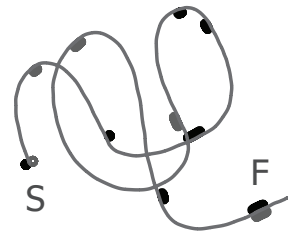

(c)

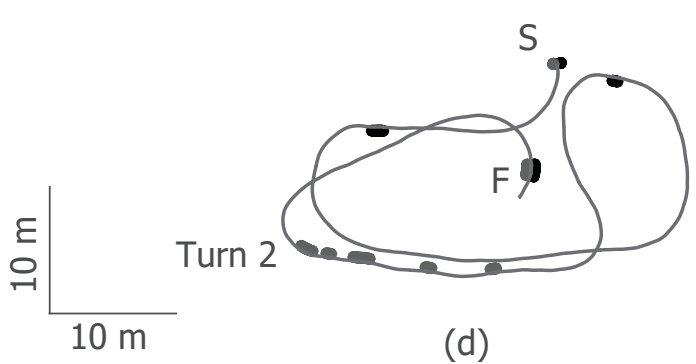

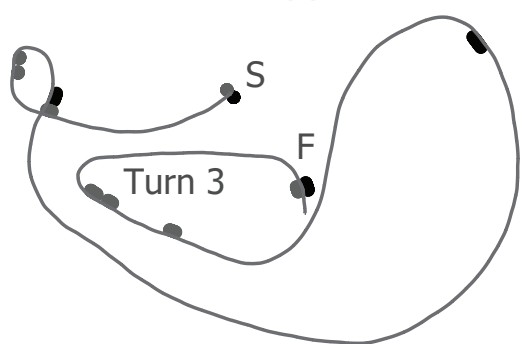

(e)

Figure 4. Trajectories of horseback riding. (a-c) show the estimated trajectory completed by the control subject. Panels (d-e) show the trajectories carried out by the deafblind rider. The gray and black makers indicate vibration of the right and left actuator, respectively. 


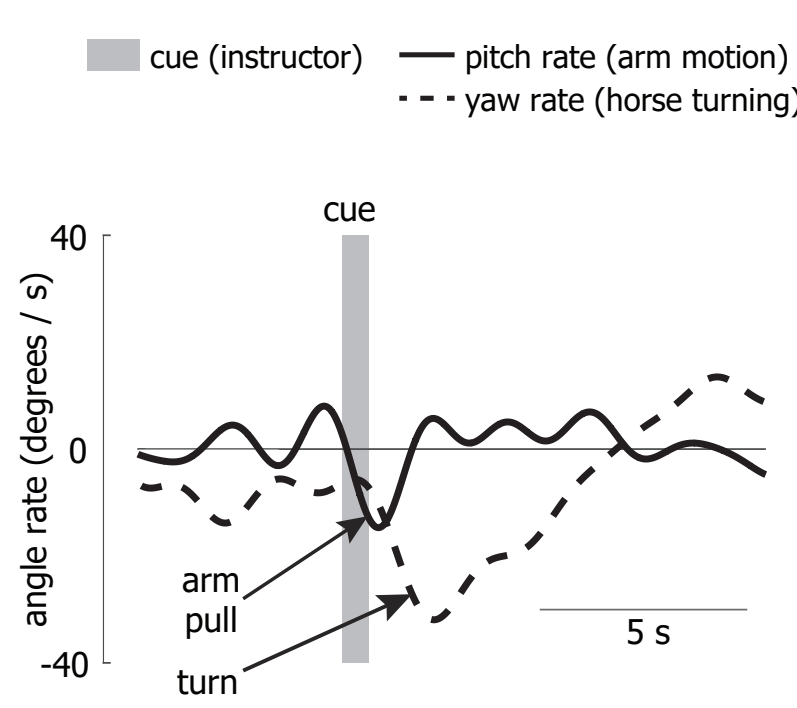

(a) Turn 1 (control)

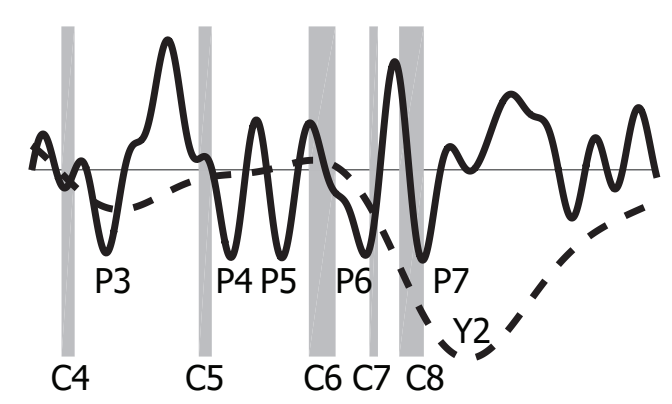

(b) Turn 2

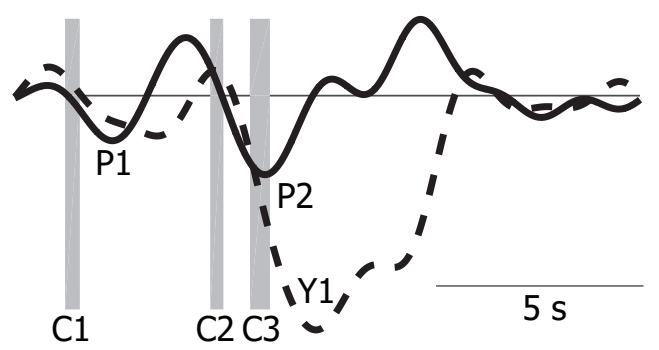

(c) Turn 3

Figure 5. Examples of the rate of change of pitch and yaw in response to tactile cues to turn right. The pitch rate indicates motion of the arm, yaw represents the orientation of the horse

the deafblind subject. One can observe that the horse started changing the direction only after the third and fifth cue in case of turns 2 and 3 respectively. However, rider's pulling in response to initial cues can be observed. Furthermore, responses P4 and P5 following the cue C5 suggest that the rider repeated the command after realizing the horse did not respond to the initial command. Overall the deafblind subject pulled the reins in response to all cues instructing him to turn the horse. Some of this, however, did not result in a turn of the horse.

We analyzed the riders' responses to tactile cues in terms of change of orientation (yaw) during riding. We considered the yaw angle starting from the instance when the stimulus occurred and lasting 10 seconds. If another instruction to turn in the opposite direction occurred during the 10 seconds, the analyzed sample was terminated. Fig. 6 depicts the distributions of riders' executed turns gathered from all trials. The bold line shows the mean and is only computed for the duration of the shortest response. The maximum change of motion direction $\Delta \gamma$ was $122.73 \pm 34.33^{\circ}$ (mean \pm std), $136.26 \pm$ $39.53^{\circ}$. The rise time (the time at which $\Delta \gamma$ reaches $95 \%$ of its maximum) were and $7.28 \pm 1.49 \mathrm{~s}, 6.49 \pm 1.19 \mathrm{~s}$ for control and deafblind riders, respectively. The distribution of the compared data is shown in Fig. 6b,c. A two-sample t-test test did not detect a significant difference of yaw and rise time samples ( $p=0.378$ and $p=0.197$, respectively).

Questionnaire results. Table 2 lists the answers to question for the case study subject, his family and the instructor. Even though the subject found the horse follows his commands (question 1), the instructor stated that this is only somewhat true (question 15). However, according to the instructor, the rider is responding to the tactile cues, which 


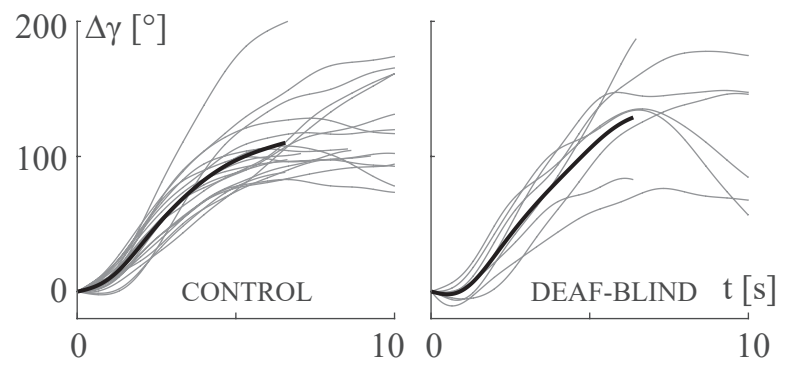

(a)

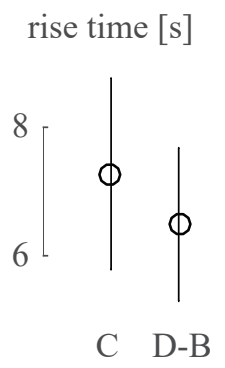

(b)

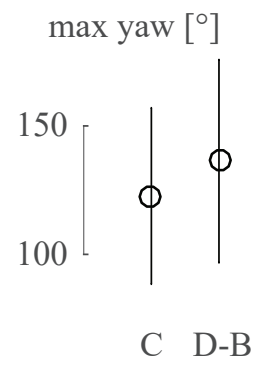

(c)

Figure 6. Analysis of orientation change elicited by the riders. (a) Orientation changes in response to a cue (gray curves) and the mean curve (thick black). The left and the right panels show the results of the control and the disabled riders, respectively. (b,c) plot the distribution of rise times and maximum yaws in the two subjects.

confirms the results obtained by means of motion capture.

The subject stated that he feels safe and comfortable while using the interface. He also indicated he does not at all find the vibrations unpleasant. The instructor did not observe any signs of the vibrations making the subject uncomfortable. However, the instructor suggested improvements of the ergonomics of the interface as the wires connecting the actuators and the control module make the attachment somewhat inconvenient.

The rider was asked to express his preference between the conventional and remote communication (questions 7 and 8 respectively). When asked which communication approach makes him feel more independent, he expressed his strong preference towards using the wireless haptic interface.

The subject strongly agreed that during use of our interface he is having more fun. In two separate questions asking whether he enjoys riding with each communication approach, the subject answered "somewhat" and "very much" for the case of conventional and remote, respectively, confirming the preference towards the remote haptic interface. The family and the instructor also observed subject's greater enjoyment and preference for the proposed interface.

Questions 18-20 and 23-25 addressed to the instructor and the family were concerned with long term therapeutic effects on the rider. The instructor's response indicated that the rider's attention span improved since the introduction of the haptic interface.

\section{Discussion}

While the control subject was $100 \%$ successful in executing the turns, the deafblind subject sometimes failed to make the horse change the direction. This quantitative result agrees with observations by the instructor reported in the questionnaires. The motion capture data analysis showed that the subject responds to the cues by pulling of the reins. The instructor also observed rider's pulls in response to cues. This observation is likely a consequence of inobedience of the horse, rider's insufficient riding skills and deficit in assertiveness. However, in the interview the subject strongly agreed with the statement that the horse follows his commands. This contradiction with motion capture data and feedback from the instructor could indicate subject's perceptual difficulty with the vestibular sense, 


\begin{tabular}{|c|c|c|}
\hline & Question & Answer \\
\hline 1 & Does the horse follow your commands? & very much \\
\hline 2 & Does riding make you comfortable? & very much \\
\hline 3 & Do you feel safe during riding? & very much \\
\hline 4 & Did you enjoy sessions with CC? & somewhat \\
\hline 5 & Did you enjoy sessions with HC? & very much \\
\hline 6 & Do you prefer $\mathrm{HC}$ or $\mathrm{CC} ?^{*}$ & HC strongly \\
\hline 7 & Did you feel more independent during $\mathrm{HC}$ or $\mathrm{CC}$ * & HC strongly \\
\hline 8 & Did you feel more confident during $\mathrm{HC}$ or $\mathrm{CC} ?^{*}$ & HC strongly \\
\hline 9 & Do you find the vibrations unpleasant? & not at all \\
\hline 10 & Was riding more fun using $\mathrm{CC}$ or $\mathrm{HC}$ * & HC strongly \\
\hline \multicolumn{3}{|c|}{ (a) Rider } \\
\hline 11 & Does the rider seem to be enjoying the riding? & very much \\
\hline 12 & Does he seem to prefer $\mathrm{HC}$ or $\mathrm{CC}{ }^{*}$ & HC strongly \\
\hline 13 & Does he seem to find the vibrations unpleasant? & not really \\
\hline 14 & Does he respond to all vibration cues? & very much \\
\hline 15 & Does the horse follow his commands? & somewhat \\
\hline 16 & Does he seem enthusiastic about riding before each session? & very much \\
\hline 17 & Does he seem more enthusiastic since introduction of HC? & very much \\
\hline 18 & Does he have a short attention span and is easily distracted? & somewhat \\
\hline 19 & If yes, has his attention span improved since introduction of CC? & somewhat \\
\hline \multicolumn{3}{|c|}{ (b) Instructor } \\
\hline 21 & Does he seem to be enjoying the riding? & very much \\
\hline 22 & Does he seem to prefer $\mathrm{HC}$ or $\mathrm{CC} ?^{*}$ & HC strongly \\
\hline 23 & Does he have short attention span and is easily distracted? & undecided \\
\hline 24 & Is his attention span and focus improved since introduction of CC? & undecided \\
\hline \multicolumn{3}{|c|}{$\begin{array}{ll}\text { (c) Family } & \end{array}$} \\
\hline Tab & & \\
\hline Que & ionnaires filled by the rider, instructor and rider's family & arked with \\
\hline
\end{tabular}

causing the subject to not be well aware of the change of orientation as a result of making the turn. However, the motion capture results do not support this reasoning. Following cue C5, the subject responded by pulling of the reins which did not result in a turn. The subject seems to have detected that and repeated his command. Finally, no issues with balance have been reported previously.

The qualitative results suggest that the proposed interface brings the user a greater sense of independence and joy. The interface does not cause the subject a sensation of discomfort or being unsafe. Instructor's observation that the rider's attention span improved since the introduction of the haptic interface is encouraging, however, the authors recognize that the result is likely subjected to bias as the duration of the conventional therapy prior to introduction of the remote interface was much shorter. 


\section{Conclusion}

We presented the first haptic interface which enables deafblind people to practice horse riding independently of the direct proximity of a riding instructor. A deafblind rider is able to receive navigation instructions wirelessly via tactile stimulation. In this paper we presented a case study involving an autistic deafblind rider. We showed that the subject was able to perceive and carry out riding commands similarly as an unimpaired blindfolded subject. Additionally, we investigated the benefits of the interface compared to conventional riding for the deafblind. Our proposed approach was preferred by the subject, as it is providing the rider with the sense of independence and bring him joy. The value of these perceptions lies in the resulting motivation to practice horseback riding, which may in turn result in other positive effects on his wellbeing. In the future we would like to introduce the interface to a larger number of users. The ergonomics could be improved by alternative placement of the actuators and/or integration into a garment.

\section{References}

American Psychiatric Association. (2000). Diagnostic and statistical manual of mental disorders DSM-IV-TR fourth edition (text revision). Washington, DC: American Psychiatric Association.

Azenkot, S., Prasain, S., Borning, A., Fortuna, E., Ladner, R. E., \& Wobbrock, J. O. (2011). Enhancing independence and safety for blind and deaf-blind public transit riders. In Proceedings of the sigchi conference on human factors in computing systems (pp. 3247-3256). ACM.

Baranek, G. T., David, F. J., Poe, M. D., Stone, W. L., \& Watson, L. R. (2006). Sensory experiences questionnaire: Discriminating sensory features in young children with autism, developmental delays, and typical development. Journal of Child Psychology and Psychiatry, 47(6), 591-601.

Bass, M. M., Duchowny, C. A., \& Llabre, M. M. (2009). The effect of therapeutic horseback riding on social functioning in children with autism. Journal of autism and developmental disorders, $39(9), 1261-1267$.

Blakemore, S.-J., Tavassoli, T., Calò, S., Thomas, R. M., Catmur, C., Frith, U., \& Haggard, P. (2006). Tactile sensitivity in asperger syndrome. Brain and cognition, 61(1), 5-13.

Busch, C., Tucha, L., Talarovicova, A., Fuermaier, A. B., Lewis-Evans, B., \& Tucha, O. (2016). Animal-assisted interventions for children with attention deficit/hyperactivity disorder a theoretical review and consideration of future research directions. Psychological Reports, 118(1), $292-331$.

Caporusso, N. (2008). A wearable malossi alphabet interface for deafblind people. In Proceedings of the working conference on advanced visual interfaces (pp. 445-448). ACM.

Caporusso, N., Trizio, M., \& Perrone, G. (2014). Pervasive assistive technology for the deaf-blind need, emergency and assistance through the sense of touch. In A. Holzinger, M. Ziefle, \& C. Röcker (Eds.), Pervasive health (p. 289-316). Springer London.

Carvill, S. (2001). Sensory impairments, intellectual disability and psychiatry. Journal of Intellectual Disability Research, 45(6), 467-483.

Cascio, C., McGlone, F., Folger, S., Tannan, V., Baranek, G., Pelphrey, K. A., \& Essick, G. (2008). Tactile perception in adults with autism: a multidimensional psychophysical study. Journal of autism and developmental disorders, 38(1), 127-137.

Cass, H. (1998). Visual impairment and autism current questions and future research. Autism, 2(2), 117-138.

Changeon, G., Graeff, D., Anastassova, M., \& Lozada, J. (2012). Tactile emotions: a vibrotactile tactile gamepad for transmitting emotional messages to children with autism. In International 
conference on human haptic sensing and touch enabled computer applications (pp. 79-90). Springer.

Dakopoulos, D., \& Bourbakis, N. G. (2010). Wearable obstacle avoidance electronic travel aids for blind: a survey. IEEE Transactions on Systems, Man, and Cybernetics, Part C (Applications and Reviews), 40(1), 25-35.

Dammeyer, J. (2011). Mental and behavioral disorders among people with congenital deafblindness. Research in developmental disabilities, 32(2), 571-575.

Dammeyer, J. (2012). Identification of congenital deafblindness. British Journal of Visual Impairment, 30(2), 101-107.

Dammeyer, J. (2014). Deafblindness: A review of the literature. Scandinavian journal of public health.

Drescher, L. (2006). Recognition of deafblindness in the European Union. Retrieved August 1st 2016, from http://www.sense.org.uk/sites/default/files/EDbN_survey_report _english_0.pdf

Edwards, N. E., \& Beck, A. M. (2002). Animal-assisted therapy and nutrition in Alzheimer's disease. Western Journal of Nursing Research, 24 (6), 697-712.

Fine, A. H. (2010). Handbook on animal-assisted therapy: Theoretical foundations and guidelines for practice. Academic Press.

Gollner, U., Bieling, T., \& Joost, G. (2012). Mobile lorm glove: introducing a communication device for deaf-blind people. In Proceedings of the sixth international conference on tangible, embedded and embodied interaction (pp. 127-130). ACM.

Güçlü, B., Tanidir, C., Mukaddes, N. M., \& Ünal, F. (2007). Tactile sensitivity of normal and autistic children. Somatosensory 83 motor research, 24(1-2), 21-33.

Kaga, K., Shinjo, Y., Jin, Y., \& Takegoshi, H. (2008). Vestibular failure in children with congenital deafness. International journal of audiology, 47(9), 590-599.

Kern, J. K., Garver, C. R., Grannemann, B. D., Trivedi, M. H., Carmody, T., Andrews, A. A., \& Mehta, J. A. (2007). Response to vestibular sensory events in autism. Research in Autism Spectrum Disorders, 1(1), 67-74.

Kern, J. K., Trivedi, M. H., Garver, C. R., Grannemann, B. D., Andrews, A. A., Savla, J. S., ... Schroeder, J. L. (2006). The pattern of sensory processing abnormalities in autism. Autism, $10(5), 480-494$.

Maclean, N., Pound, P., Wolfe, C., \& Rudd, A. (2002). The concept of patient motivation a qualitative analysis of stroke professionals' attitudes. Stroke, 33(2), 444-448.

Moller, C. (2003). Deafblindness: living with sensory deprivation. The Lancet, 362, s46-s47.

Nagel, S. K., Carl, C., Kringe, T., Märtin, R., \& König, P. (2005). Beyond sensory substitution learning the sixth sense. Journal of neural engineering, 2(4), R13.

O'Haire, M. E. (2013). Animal-assisted intervention for autism spectrum disorder: A systematic literature review. Journal of autism and developmental disorders, 43(7), 1606-1622.

Puts, N. A., Wodka, E. L., Tommerdahl, M., Mostofsky, S. H., \& Edden, R. A. (2014). Impaired tactile processing in children with autism spectrum disorder. Journal of Neurophysiology, $111(9), 1803-1811$.

Richeson, N. E. (2003). Effects of animal-assisted therapy on agitated behaviors and social interactions of older adults with dementia. American journal of Alzheimer's disease and other dementias, 18(6), 353-358.

Sabatini, A. M., Martelloni, C., Scapellato, S., \& Cavallo, F. (2005). Assessment of walking features from foot inertial sensing. Biomedical Engineering, IEEE Transactions on Biomedical Engineering, 52(3), 486-494.

Sams, M. J., Fortney, E. V., \& Willenbring, S. (2006). Occupational therapy incorporating animals for children with autism: A pilot investigation. American Journal of Occupational Therapy, $60(3), 268-274$.

Scheggi, S., Talarico, A., \& Prattichizzo, D. (2014). A remote guidance system for blind and visually 
impaired people via vibrotactile haptic feedback. In Control and automation (med), 2014 22nd mediterranean conference of (pp. 20-23). IEEE.

Tang, F., McMahan, R. P., \& Allen, T. T. (2014). Development of a low-cost tactile sleeve for autism intervention. In Haptic, audio and visual environments and games (have), 2014 ieee international symposium on (pp. 35-40). IEEE.

Tsukada, K., \& Yasumura, M. (2004). Activebelt: Belt-type wearable tactile display for directional navigation. In Ubicomp 2004: Ubiquitous computing (pp. 384-399). Springer.

World Health Organisation. (2014). Visual impairment and blindness. Retrieved 2016-01-24, from http://www . who.int/mediacentre/factsheets/fs282/en/ 